\title{
Revista Colombiana de

\section{Mixoma auricular izquierdo como causa de accidente cerebral isquémico transitorio e infarto agudo de miocardio con elevación del ST}

\author{
Manuel J. Liévano ${ }^{a}$, Jorge Sánchez $\mathrm{C}^{\mathrm{a}, *}$, Gabriel Acosta $\mathrm{S}^{\mathrm{a}}$, \\ Jennifer Cifuentes $T^{a}$, Héctor Olaya $R^{a}$ y Sara Ramírez $A^{b}$ \\ a Hemodinamia y Cardiología Intervencionista, Hospital de San José, Bogotá, Colombia \\ b Cardiología, Clínica Los Nogales, Bogotá, Colombia
}

Recibido el 3 de marzo de 2019; aceptado el 5 de noviembre de 2019

Disponible en Internet el 21 de abril de 2020

\section{PALABRAS CLAVE \\ Mixoma; \\ Infarto agudo de \\ miocardio; \\ Infarto cerebral; \\ Angioplastia; \\ Angiografía; \\ Trombólisis}

\section{KEYWORDS}

Myxoma;

Acute myocardial

infarction;

Stroke;

Angioplasty;

Angiography;

Thrombolysis

\begin{abstract}
Resumen Los mixomas son los tumores cardíacos primarios más comunes. La mayoría tiene curso benigno y pueden tratarse de hallazgos incidentales o ser casi asintomáticos. Los síntomas dependen de su ubicación, en cuyo caso la más frecuente es la aurícula izquierda, de ahí que el riesgo embólico sea elevado. El principal lugar de embolia es el sistema nervioso central; sin embargo, en ocasiones puede afectar los sistemas respiratorio, periférico o coronario ${ }^{1}$.

Se expone el caso de una paciente con mixoma auricular izquierdo, el cual se manifiesta con accidente cerebral isquémico transitorio, seguido de infarto agudo de miocardio con elevación del segmento ST en la pared ínfero-posterior.

(C) 2019 Sociedad Colombiana de Cardiología y Cirugía Cardiovascular. Publicado por Elsevier España, S.L.U. Este es un artículo Open Access bajo la licencia CC BY-NC-ND (http:// creativecommons.org/licenses/by-nc-nd/4.0/).
\end{abstract}

Left atrial myxoma as a cause of a transient ischaemic stroke and acute myocardial infarction with ST elevation

\begin{abstract}
The myxomas are the most common primary cardiac tumours. The majority of them are benign and can be an incidental finding or be almost asymptomatic. The symptoms depend on their location, with the most common being the left atrium, which carries an increased risk of clots. The main effects of the clots are on the central nervous system, but occasionally they can involve the respiratory, peripheral, or coronary system.
\end{abstract}

\footnotetext{
* Autor para correspondencia.

Correo electrónico: jorgesanchezcantillo@gmail.com (J.S. C).
} 
The case is presented of a patient with left atrial myxoma, as well as a transient ischaemic stroke and acute myocardial infarction with ST elevation in the inferior-posterior wall.

(c) 2019 Sociedad Colombiana de Cardiología y Cirugía Cardiovascular. Published by Elsevier España, S.L.U. This is an open access article under the CC BY-NC-ND license (http:// creativecommons.org/licenses/by-nc-nd/4.0/).

\section{Introducción}

Los tumores cardíacos tienen muy baja incidencia, aproximadamente $0,1 \%$, y entre estos los metastásicos son los más comunes. En cuanto a los tumores benignos, en el $50 \%$ de los casos se trata de mixomas, que son los tumores cardíacos primarios más comunes en los adultos. Pueden ubicarse en cualquier parte del corazón; sin embargo, su localización más frecuente es la aurícula izquierda ${ }^{1}$. Los síntomas dependen de su infiltración y localización. Se presenta el caso de una paciente de 63 años, con un mixoma en la aurícula izquierda, de aproximadamente $3 \mathrm{~cm}^{2}$, que se manifiesta inicialmente con un accidente cerebral isquémico transitorio y es seguido de un síndrome coronario agudo.

\section{Caso}

Paciente de 63 años de edad, natural de La Palma, Cundinamarca, residente en Bogotá, operadora en un supermercado, quien consultó por disartria y cefalea de 10 minutos de duración, de resolución espontánea, sin dejar focalización neurológica. Como antecedentes patológicos describió dislipidemia e hipotiroidismo. En la revisión por sistemas llamó la atención el deterioro de clase funcional de II a III/IV en la escala de la New York Heart Association (NYHA) de 2 meses de evolución. Durante el examen físico se encontraron signos vitales normales, sobrepeso, sin agregados pulmonares y ruidos cardíacos normales. Fue valorada en el servicio de urgencias del Centro Policlínico del Olaya (CPO), donde realizaron tomografía axial computarizada de cráneo simple, la cual estuvo dentro de límites normales; doppler de vasos de cuello normal y ecocardiograma transtorácico que documentó una masa móvil en la aurícula izquierda. En consecuencia, se ordenó un ecocardiograma transesofágico (fig. 1A) que reportó una masa lobulada en la aurícula izquierda, de ecogenicidad intermedia, de aproximadamente $2,0 \times 17 \mathrm{~mm}$, con pedículo sobre el septum interauricular, aproximadamente a $2 \mathrm{~cm}$ del plano valvular mitral, sin obstrucción al tracto de salida del ventrículo izquierdo; ventrículo izquierdo de forma y tamaño normal, sin alteraciones en la contractilidad y fracción de eyección del ventrículo izquierdo (FEVI) del 68\%.

Durante la hospitalización en el CPO presentó dolor torácico típico en horas de la madrugada, con evidencia electrocardiográfica de elevación del segmento ST en cara ínfero-posterior (fig. 1B). Consideraron contraindicación para trombólisis por antecedente de accidente cerebral isquémico transitorio y decidieron remisión a la Clínica Los Nogales, institución de mayor complejidad.
Ingresó a dicha entidad en horas de la madrugada, con signos vitales inestables (hipotensión arterial) y persistencia de angina, electrocardiograma de control con mayor supradesnivel del segmento ST, por lo que decidieron trombólisis farmacológica con tenecteplase, luego de la cual, por persistencia de angina, fue llevada a intervención coronaria percutánea de rescate (figs. $1 \mathrm{C}$ y D).

En la arteriografía coronaria se evidenció una arteria descendente anterior con placas no significativas en el tercio medio y oclusión distal del vaso; primera arteria diagonal con lesión proximal del $90 \%$; arteria circunfleja con oclusión total del vaso en el tercio medio, con abundante trombo; y arteria coronaria derecha sin lesiones (fig. 2). Se realizó angioplastia con balón convencional de 3,0 x $15 \mathrm{~mm}$ de la oclusión total de la arteria circunfleja, con recanalización de la luz del vaso y evidencia de abundante trombo, posterior tromboaspiración con catéter y administración de tirofibán intracoronario e infusión por 24 horas. La angiografía final mostró discreta resolución en la carga del trombo y flujo TIMI I (escala del flujo sanguíneo epicárdico) (fig. 2A).

Un nuevo ecocardiograma transtorácico de control luego de 72 horas postevento coronario documentó FEVI del 35\%, aquinesia de las paredes inferior y posterior, así como masa pediculada de la aurícula izquierda de $3,5 \mathrm{~cm}^{2}$ (fig. 2B).

El caso se presentó en junta médico-quirúrgica de la institución con el fin de determinar la necesidad de revascularización de la arteria circunfleja y emitir consenso acerca de la realización de una perfusión miocárdica con protocolo para viabilidad, que evidenció necrosis del $25 \%$ en territorio de la arteria circunfleja y de la pared inferior; finalmente, se decidió solo hacer la resección quirúrgica de la masa (fig. 3).

La paciente evolucionó de forma satisfactoria en el posoperatorio y fue dada de alta a los 8 días de su ingreso, sin complicaciones.

Se valoró en consulta al mes posoperatorio en la que no se observaron secuelas neurológicas, y permanecía en clase funcional I de la NYHA, sin complicaciones. El reporte de Patología confirmó mixoma sin elementos glandulares, e inmunohistoquímica con calretinina $(+)$, CD31 (+), CD $34(+)$, S100 (+), vimentina (+), CKAE1AE3 (-), CD68 (+) y Ki67: 4\%.

\section{Discusión}

La incidencia de tumores cardíacos es baja y la mayoría de éstos, aproximadamente del 50 al $80 \%$, son mixomas ${ }^{1}$. Tienen buen pronóstico a largo plazo, incluso en resecciones incompletas. Pueden tratarse de hallazgos incidentales o ser sintomáticos; los signos y síntomas específicos dependerán de la localización y el tamaño, mas no de la histopatología. 

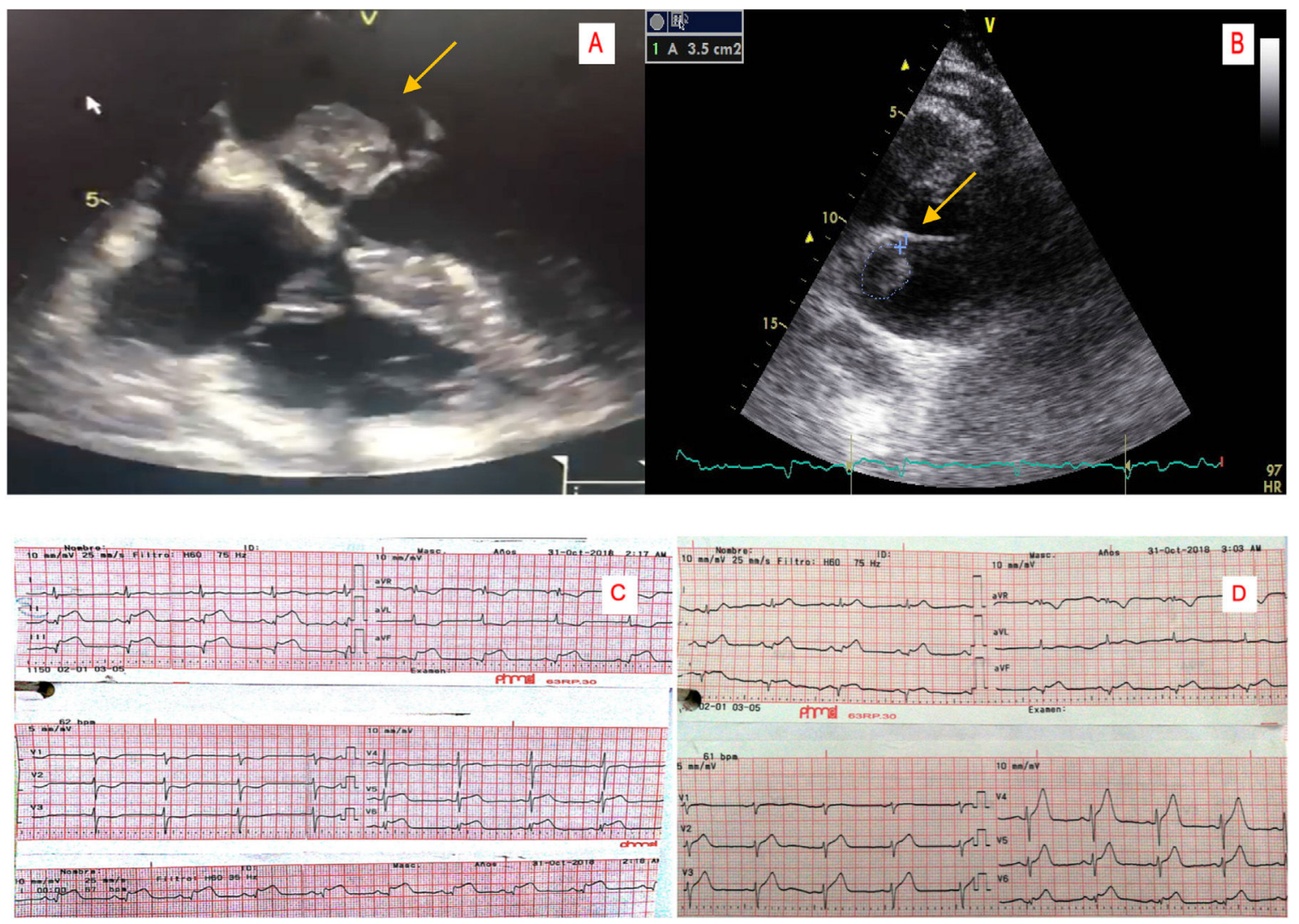

Figura 1 A. Electrocardiograma que muestra lesión subepicárdica en la pared inferior y lateral. B. Electrocardiograma postrombólisis en el que se evidencia mejoría del supradesnivel del segmento ST. C. Imagen del mixoma en el ecocardiograma transesofágico. D. Imagen del mixoma en ecocardiograma transtorácico.

Aproximadamente, el $80 \%$ se ubican en la aurícula izquierda. Adicional a los síntomas cardiovasculares es posible encontrar síntomas constitucionales debido a la producción de citoquinas y de factores de crecimiento, o también es posible hallar síntomas extracardíacos ${ }^{2}$.

La presentación clínica es variable; puede producir síntomas por interferencia mecánica con la función cardíaca y causar congestión pulmonar o, cuando se fragmentan, embolias sistémicas. Los síntomas cardíacos dependerán del tamaño del tumor, por ejemplo síntomas de falla cardiaca por obstrucción de la válvula mitral o hallazgos de hipertrofia de aurícula izquierda en el electrocardiograma ${ }^{2}$.

La embolización es una manifestación común, la cual se asocia con tumores de pequeño tamaño $\left(<4,5 \mathrm{~cm}^{2}\right)$. La mayoría de los émbolos migra al sistema nervioso central y causa accidentes cerebrovasculares; sin embargo, pueden migrar a cualquier parte del sistema arterial y producir variedad de signos y síntomas. Hay casos reportados de embolia a miembros inferiores o a las coronarias, entre otros. En algunos casos puede haber manifestaciones cardíacas y extracardíacas, incluyendo infarto agudo de miocardio, eventos cerebrovasculares, embolia pulmonar y fiebre de origen desconocido; no obstante, se ha establecido una tríada clásica, que consiste en síntomas obstructivos y constitucionales, además de eventos embólicos. En el caso expuesto, durante la misma hospitalización se registraron dos manifestaciones extracardiacas, dadas por episodio de accidente cerebral isquémico transitorio e infarto de miocardio con elevación del ST. Similar a este caso hay otro reportado previamente en la literatura, en el que un paciente sin factores de riesgo presentó inicialmente síndrome coronario agudo, seguido de accidente cerebrovascular ${ }^{3}$.

El diagnóstico es un desafío; se realiza a través de ecocardiograma transtorácico, transesofágico y resonancia nuclear magnética cardiaca, aunque en ocasiones se puede identificar mediante tomografía axial computarizada cardiaca. El ecocardiograma puede visualizar fácilmente la masa y describir ubicación, forma, tamaño, número y características morfológicas; además, valora las consecuencias hemodinámicas del tumor ${ }^{4}$. En la tomografía, los mixomas pueden ser bien definidos, aparecer lobulados, lisos, móviles, redondos u ovales, con un pedículo estrecho; generalmente son heterogéneos y tienen focos de calcificación y realce en parches $^{5}$.

En cuanto al tratamiento, se requiere experticia y siempre será quirúrgico. Es importante determinar el origen y la malignidad de estos tumores en el preoperatorio. Un ejemplo bien conocido es el carcinoma renal con expansión a la aurícula derecha, que puede confundirse con un tumor primario antes de llevar a cirugía. Adicionalmente, no todos son tumores reales pues existen los "pseudotumores", los trombos, los quistes y los tuberculomas ${ }^{5}$.

Se deben evaluar los vasos venosos superficiales de los miembros inferiores en caso de posible reconstrucción auricular y revascularización miocárdica, o ambas, así como 

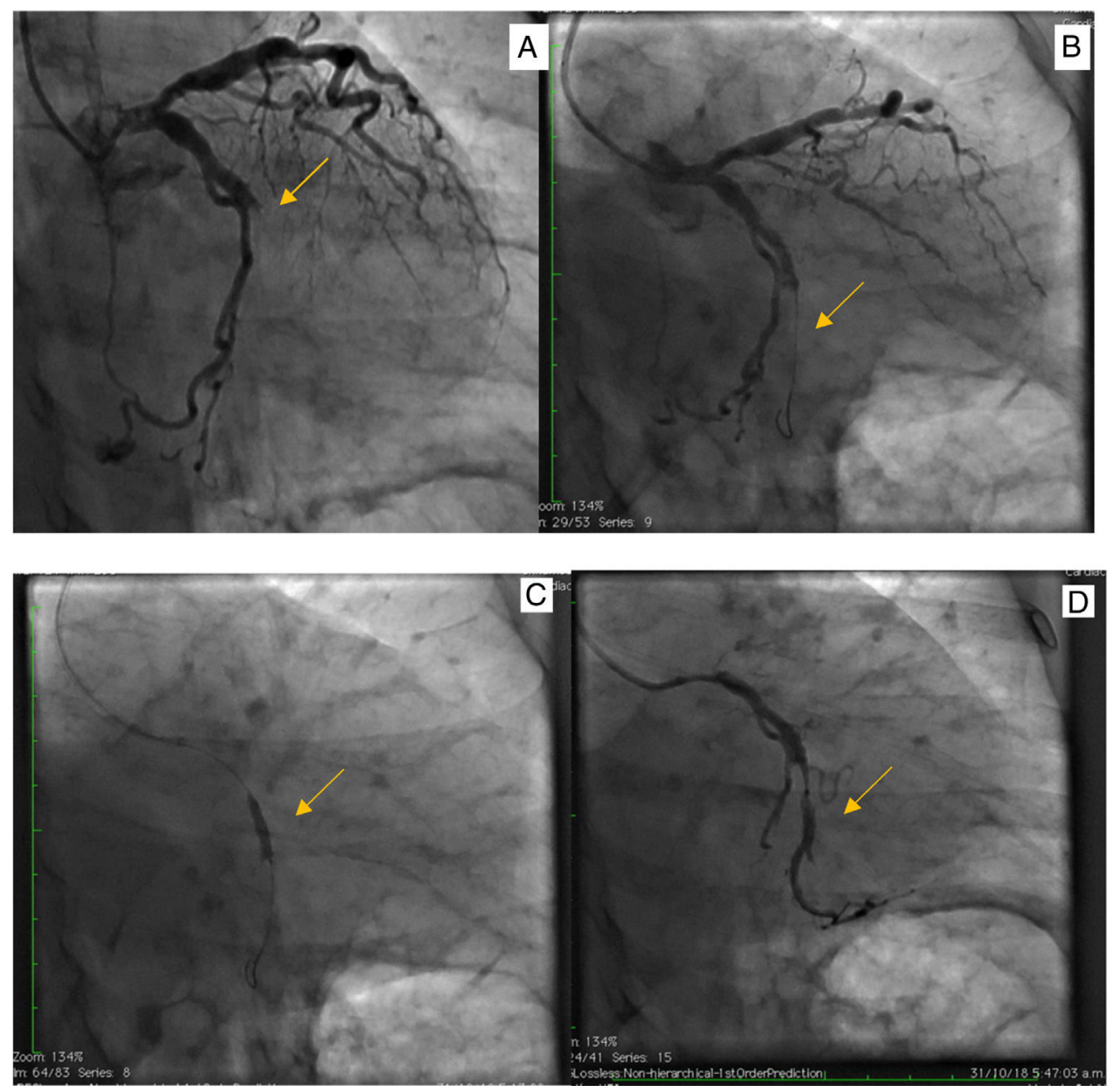

Figura 2 A. Arteriografía coronaria en proyección oblicua derecha con caudal con oclusión del 100\% de la arteria circunfleja. B. Paso de guía de angioplastia coronaria 0,014 '”. C. Angioplastia con balón, D. Tromboaspiración con catéter y posterior inyección control con medio de contraste que evidencia flujo anterógrado de la arteria circunfleja muy discreto TIMI 0-I.
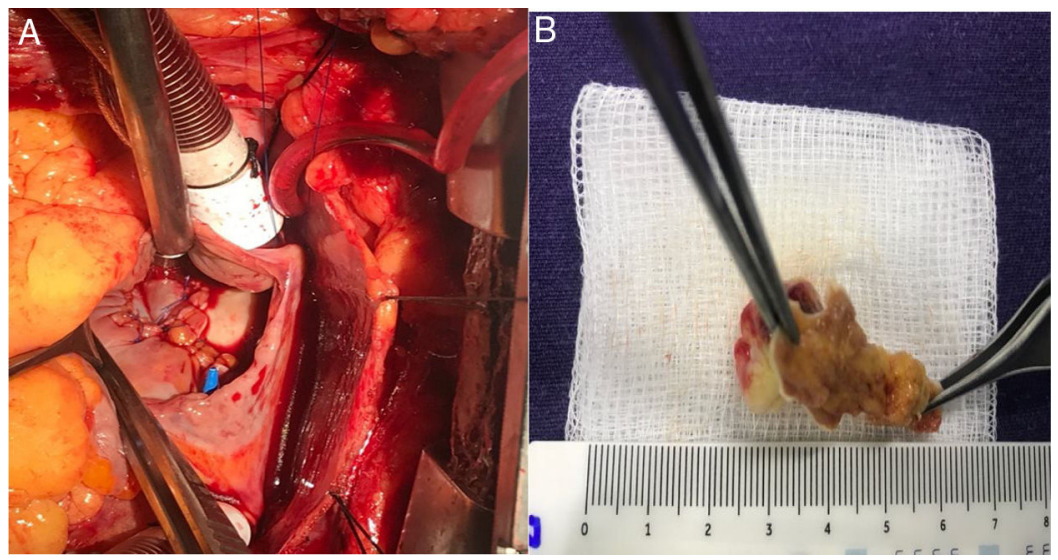

Figura 3 A. Fotografía de la cirugía cardiovascular para extirpación de mixoma auricular; vista superior de esternotomía con exposición del corazón y tubo anillado de circulación extracorpórea. B. Pieza quirúrgica obtenida.

evaluar el saco pericárdico en busca de metástasis, ya que si se observan lesiones irresecables, irreconstruibles o efusión sanguinolenta secundaria no debería continuarse el procedimiento y en ese caso pensar en métodos paliativos, al igual que si es un tumor sólido primario del pericardio en su mayoría son malignos e incurables (mesotelioma y sarcomas) ${ }^{6}$.

Las aurículas con afectación tumoral son un sitio de amplia reconstrucción, ya que la pérdida de la contractilidad auricular puede ser bien tolerada por el paciente. El 
principal tumor, ya sea en la aurícula derecha o izquierda o septum interaricular, es el mixoma y se recomienda una resección en "dona" alrededor, para verificar la resección completa de los bordes. En tumores malignos se deben descartar metástasis previas ya que si se lleva a procedimiento de resección con enfermedad a distancia la mortalidad es alta. La sobrevida libre de enfermedad con los mixomas es buena, a menos que curse con síndrome de Carney. El seguimiento se hace a los 6 meses, al año y después cada año, con ecocardiograma transtorácico ${ }^{7}$.

Respecto al uso de radioterapia o quimioterapia en estos pacientes es realmente muy bajo debido a la alta tasa de resecciones exitosas, por consiguiente se reserva solo para casos de múltiples mixomas cardíacos en localización cerebral $^{8}$.

El vaso culpable del infarto agudo del miocardio secundario a un mixoma que emboliza, generalmente es la arteria coronaria derecha, debido a la localización caudal de la arteria, que permite el paso de detritus o trombos de la superficie del tumor; sin embargo, no es inusual encontrar en la literatura reportes de casos con embolia a otros territorios coronarios, como a la arteria descendente anterior y la arteria circunfleja ${ }^{9,10}$.

En cuanto al manejo del infarto agudo del miocardio secundario a embolia por mixoma cardíaco, está descrito el tratamiento convencional en guías según el caso, que incluye manejo farmacológico con oxígeno, anticoagulación, doble antiagregación, trombólisis farmacológica, betabloqueadores y estratificación invasiva temprana con arteriografia coronaria, en la que los hallazgos orientarán la realización de angioplastia con balón, tromboaspiración con catéter de los detritos embolizados y angioplastia más implante de stent; esta última tiene alto riesgo de trombosis aguda y fenómeno de no reflujo, por lo cual, si el caso lo permite, se aconseja postergar la angioplastia más stent a un segundo tiempo ${ }^{11-13}$.

En cuanto a los eventos cerebrovasculares asociados a los mixomas cardíacos, hasta en un $22 \%$ de los casos se ha observado accidente cerebrovascular, con predominancia en el sexo femenino ${ }^{1}$. El estudio de imagen ideal es la resonancia magnética nuclear cerebral dada la alta tasa de falsos negativos que arroja la tomografía axial computarizada de cráneo. Cursan con una baja tasa de mortalidad y todavía no es claro el momento ideal para la realización de la resección del tumor; pese a ello, se ha recomendando que sea pospuesta hasta cuatro semanas después del evento cerebral para así disminuir el riesgo de muerte perioperatoria $^{13}$.

Finalmente, se recomienda el seguimiento ecocardiográfico posoperatorio ya que la tasa de recurrencia del tumor después de una resección exitosa alcanza hasta entre el 4 al $7 \%^{14}$.

\section{Conclusión}

Los mixomas cardíacos son tumores cardíacos comunes, que tienen diferentes formas de presentación. Se reporta el caso de una paciente con embolia cerebral y coronaria durante la misma hospitalización, y difícil tratamiento de su enfermedad coronaria debido al alto contenido trombótico coronario, probablemente acompañado de tejido tumoral.

\section{Financiación}

Ninguna

\section{Conflictos de interés}

Ninguno.

\section{Bibliografía}

1. Lone RA, Ahanger AG, Singh S, Mehmood W, Shah S, Lone G, et al. Atrial myxoma: trends in management. Int J Health Sci (Qassim). 2008;2:141-51.

2. Neragi-Miandoab S, Kim J, Vlahakes GJ. Malignant tumours of the heart: a review of tumour type, diagnosis and therapy. Clin Oncol. 2007;19:748-56.

3. Wang Z, Chen S, Zhu M, Zhang W, Zhang H, Li H, et al. Risk prediction for emboli and recurrence of primary cardiac myxomas after resection. J Cardiothorac Surg. 2016;11:22.

4. Aguilera B, Suárez-Mier MP, García García R. Muerte súbita por embolia cerebral de mixoma de la aurícula izquierda. Cuad Med Forense. 2011;17:149-53.

5. Coley C, Lee KR, Steiner M, Thompson CS. Complete embolization of a left atrial myxoma resulting in acute lower extremity ischemia. Texas Hear Inst J. 2005;32:238.

6. Wang Q, Yang F, Zhu F, Yao C. A case report of left atrial myxoma-induced acute myocardial infarction and successive stroke. Medicine (Baltimore). 2018;97.

7. Mert KU. Unusual aspects of cardiac myxoma. Anatol J Cardiol [Internet]. 2017:241-7. Disponible en: https://www. journalagent.com/anatoljcardiol/pdfs/AJC_17_3_248.pdf.

8. Smith M, Chaudhry MA, Lozano P, Humphrey MB. Cardiac myxoma induced paraneoplastic syndromes: a review of the literature. Eur J Intern Med. 2012;23:669-73.

9. Report C. Catastrophic systemic embolization from a left atrial myxoma. 2014:64-6.

10. Hospital H. Atypical presentation of the most typical cardiac tumor. 2013:1-3.

11. Germany GH, Germany AK, Lenzen MJ, Denmark EP, Vranckx P. 2017 ESC Guidelines for the management of acute myocardial infarction in patients presenting with ST -segment elevation The Task Force for the management of acute myocardial infarction. 2017:1-66.

12. Jiao ZY, Zhang DP, Xia K, Wang LF, Yang XC. Clinical analysis of acute myocardial infarction caused by coronary embolism. J Thorac Dis. 2017;9:2898-903.

13. Thygesen K, Alpert JS, Jaffe AS, Chaitman BR, Bax JJ, Morrow DA, et al. Fourth universal definition of myocardial infarction (2018). Circulation [Internet]. 2018;(August):1. Disponible en: http://insights.ovid.com/crossref?an $=00003017$ 900000000-95028.

14. Yuan S. Cardiac myxoma: a rare cause of acute myocardial infarction. 2016;24:166-72. 